\title{
Characterization of Energetic Protons Generated in the ShenGuang-II UP Petawatt Laser Interactions with Foil Targets
}

\author{
Huiya Liu $\mathbb{D}^{1},{ }^{1}$ Anle Lei $\mathbb{D}^{1},{ }^{1,2,3}$ Ning Kang, ${ }^{1}$ Honghai An, ${ }^{2}$ Zhiyong Xie, ${ }^{2}$ Yao Zhao, ${ }^{1}$ \\ Shenlei Zhou, ${ }^{1}$ Mingying Sun, ${ }^{1}$ Baoqiang Zhu, ${ }^{1}$ Wei Wang, ${ }^{2}$ and Jianqiang Zhu ${ }^{1}$ \\ ${ }^{1}$ National Laboratory on High Power Laser and Physics, Shanghai Institute of Optics and Fine Mechanics, \\ Chinese Academy of Sciences, Shanghai 201800, China \\ ${ }^{2}$ Shanghai Institute of Laser Plasma, China Academy of Engineering Physics, Shanghai 201800, China \\ ${ }^{3}$ Center for Ultimate Energy, ShanghaiTech University, Shanghai 201210, China \\ Correspondence should be addressed to Anle Lei; lal@siom.ac.cn
}

Received 14 May 2021; Accepted 2 August 2021; Published 18 August 2021

Academic Editor: Yuji Fukuda

Copyright (c) 2021 Huiya Liu et al. This is an open access article distributed under the Creative Commons Attribution License, which permits unrestricted use, distribution, and reproduction in any medium, provided the original work is properly cited.

\begin{abstract}
The characterization of energetic protons generated in the ShenGuang-II UP petawatt laser interactions with foil targets has been systematically studied. The proton energy spectra and angular distributions are measured with a radiochromic film stack. It shows that the proton energy spectra have a Boltzmann distribution with temperature of about $2.8 \mathrm{MeV}$ and cutoff energy of about $20 \mathrm{MeV}$. The divergence angles of protons vary from $10^{\circ}$ to $60^{\circ}$, dependent on the proton energy. The proton source size and location are investigated via the proton point-projection mesh imaging. The proton virtual sources are found to locate tens to hundreds of microns in front of the foil target, depending on the proton energies. A Monte Carlo simulation estimates the diameter of the virtual proton source to be about $12 \mu \mathrm{m}$ for the protons with energy of $16.8 \mathrm{MeV}$, which is much smaller than the laser focus size of about $50 \mu \mathrm{m}$. The spatial resolution of the $16.8 \mathrm{MeV}$ proton imaging is quantified with the point spread function to be about $15 \mu \mathrm{m}$, which is consistent with the proton virtual source size. These results will be important for the users conducting experiments with the protons as a backlighting source on the ShenGuang-II UP petawatt laser.
\end{abstract}

\section{Introduction}

The energetic protons generated in short, intense laser interactions with foil targets have received much attention in recent years and have been widely studied both experimentally and theoretically [1-5]. The protons accelerated by the electric field at the rear side of the target via the target normal sheath acceleration (TNSA) mechanism have properties including short pulse duration and high brightness [6]. The protons have many potential applications, such as probing the fast dynamics of electric or magnetic fields in the laser produced plasmas [7-10], as a fast ignitor beam for laser-driven fusion [11], proton radiobiology [12-16], material science [17], and neutron production [18]. The characteristics of the laser-driven protons such as energy spectrum, divergence angle, and source size are essential for these applications.
The energetic protons accelerated through TNSA are from the water or hydrocarbon contaminant layers on the target rear surface and can be accelerated to tens of $\mathrm{MeV}$. Usually, in the point-projection imaging experiments, the size of the source decides the best resolution [19]. However, the transverse size of the proton source was measured to be much larger than the imaging resolution [20]. It was, therefore, considered that the protons were emitted in a quasilaminar fashion from a virtual source with a small size $[11,21]$.

ShenGuang-II UP laser facility at the National Laboratory on High-Power Laser and Physics is a multifunctional experimental platform, which includes eight kilojoule-class nanosecond laser beams and one picosecond petawatt laser beam (called the SG-II UP petawatt laser, i.e., the SG-II UP PW laser) [22]. The SG-II UP PW laser is usually used for fast heating of an imploded dense core plasma or generating 
energetic protons as a backlight source to investigate the plasmas produced by the nanosecond lasers. In this article, the characterization of energetic protons generated in the SG-II UP PW laser interactions with foil targets was systematically presented. The proton point-projection imaging with $\mathrm{Cu}$ meshes in combination with a radiochromic film (RCF) stack was used to characterize the protons. The proton energy spectra, angular distribution, and the virtual source size and location were obtained. The spatial resolution of the proton imaging was also analysed and compared to the virtual source size. These results and findings will be important for the potential users to know the laser status and to understand the properties of the protons so as to design their experiments.

\section{Experimental Setup}

The experiment was conducted on the SG-II UP PW laser. The laser uses a hybrid technology combining optical parametric chirped-pulse amplifier and Nd:glass chirpedpulse amplifier to generate short pulse (1-10 ps) and large energy (up to $1000 \mathrm{~J}$ ) at $1053 \mathrm{~nm} \mathrm{[22].} \mathrm{The} \mathrm{experimental}$ setup is shown in Figure 1. The laser was focused with an f/2.5 off-axis parabolic mirror to the $10 \mu \mathrm{m}$ thickness $\mathrm{Au}$ foil targets, at an incidence angle of $21^{\circ}$. In some shots, a $\mathrm{Cu}$ mesh was put behind the foil target at a distance $d=2$ $\mathrm{mm}$, as the object in the proton point-projection imaging to judge the quality of the proton imaging. The meshes (AG 200, Gilder Grids) were formed by $36 \mu \mathrm{m}$ lines with $90 \mu \mathrm{m}$ spacing. The protons emitting from the rear surface of the target passed through the mesh and were detected with RCF (HD-V2, GafChromic) stacks [23]. The front surface of the RCF stack was located $L=32 \mathrm{~mm}$ behind the $\mathrm{Cu}$ mesh.

An X-ray pinhole camera (XPHC) with the theoretical resolution of about $13 \mu \mathrm{m}$ was used to monitor the laser focus spot $[24,25]$. An imaging plate (BAS-SR, Fuji Film) was used as the $\mathrm{X}$-ray detector. In the experiment, the incident laser energy and pulse duration were set at $130 \mathrm{~J}$ and 1 ps, respectively, which represents a routine status of the laser as a proton backlighter. Figure 2(a) shows a typical XPHC image of the laser focus. Because a lot of physical processes are involved in the X-ray emission, such as fast electron reflux, recirculation, and transport inside the target (collision, Ohmic heating, etc.), scientifically scaling the measured emitted X-ray profile with the real laser focus intensity on the target is difficult. In this article, we assume that the X-ray intensity obtained by XPHC scales linearly with the laser intensity. The cumulative laser energy fraction as functions of intensity and focus diameter can be obtained, as shown in Figure 2(b). It shows that about $50 \%$ of the laser energy is contained within a $60 \mu \mathrm{m}$ diameter, resulting in laser intensity over $1.5 \times 10^{18} \mathrm{~W} / \mathrm{cm}^{2}$. The experimental results measured in each shot are shown in Figure 2(c), and the shot number 1 is chosen as a typical shot. The incident laser energies are stable at $130 \mathrm{~J} \pm 15 \%$, and the proton cutoff energies measured with the RCF stacks are all higher than $15 \mathrm{MeV}$.

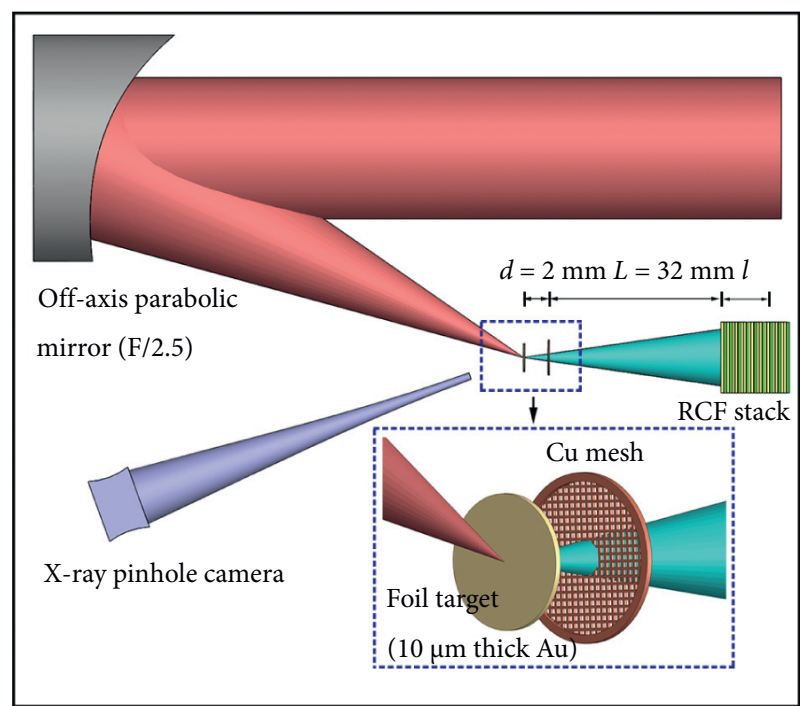

FIGURE 1: Experimental setup.

\section{Experimental Results and Analysis}

3.1. Proton Energy Spectra and Angular Distribution. The generated protons pass through the RCF layers, depositing their kinetic energy in the RCFs [26]. Typical proton images recorded on the RCFs scanned by using the EPSON V750 scanner for the same shot as in Figure 2(a) are shown in Figure 3(a). It is obvious that the lower-energy protons have higher intensity and larger angular divergence angle. The maximum proton energy reaches up to $18.9 \mathrm{MeV}$.

The energy spectrum of the protons can be obtained from the RCF images. Based on the calibration of the HDV2-type RCF for protons with different doses in [23, 26, 27], the optical density of the RCFs can be converted into the deposited proton energy. The spatially integrated proton number on each RCF layer can, thus, be obtained from the deposited energy with SRIM simulations and the spectrum unfolding calculation [28, 29], as shown in Figure 3(b). The proton energy spectrum shows an exponential profile. We fit the spectrum with the Boltzmann distribution. The fitted energy spectrum is shown in Figure 3(b), with total proton number and the temperature $k_{B} T$ of about $1.2 \times 10^{12}$ and 2.8 $\mathrm{MeV}$, respectively. As we can see from the Figure 3(b), because of the saturation of the lowest-energy RCF layers, the measured proton numbers at low proton energies are less than the fitted result. Based on the fitting result, the conversion efficiency of laser energy to the proton beam can be calculated to be about $0.62 \%$.

With the given distances between the target and the RCF layers and sizes of the proton images, the divergence angles (referring the full beam aperture in this article) of the energy-resolved protons can be calculated, as shown in Figure 3(c). The results from VULCAN and 100TW-LULI lasers by Nürnberg et al. [26] are also shown for comparison. In Figure 3(c), the proton energy $E$ is scaled to the maximum proton energy $E_{\max }$, here $E_{\max }=18.9 \mathrm{MeV}, 29.7 \mathrm{MeV}$, and 16.2 MeV for the SG-II UP PW, VULCAN, and 100TWLULI lasers, respectively. The divergence angles of protons in our experiment are from $10^{\circ}$ to $60^{\circ}$, dependent on the proton 

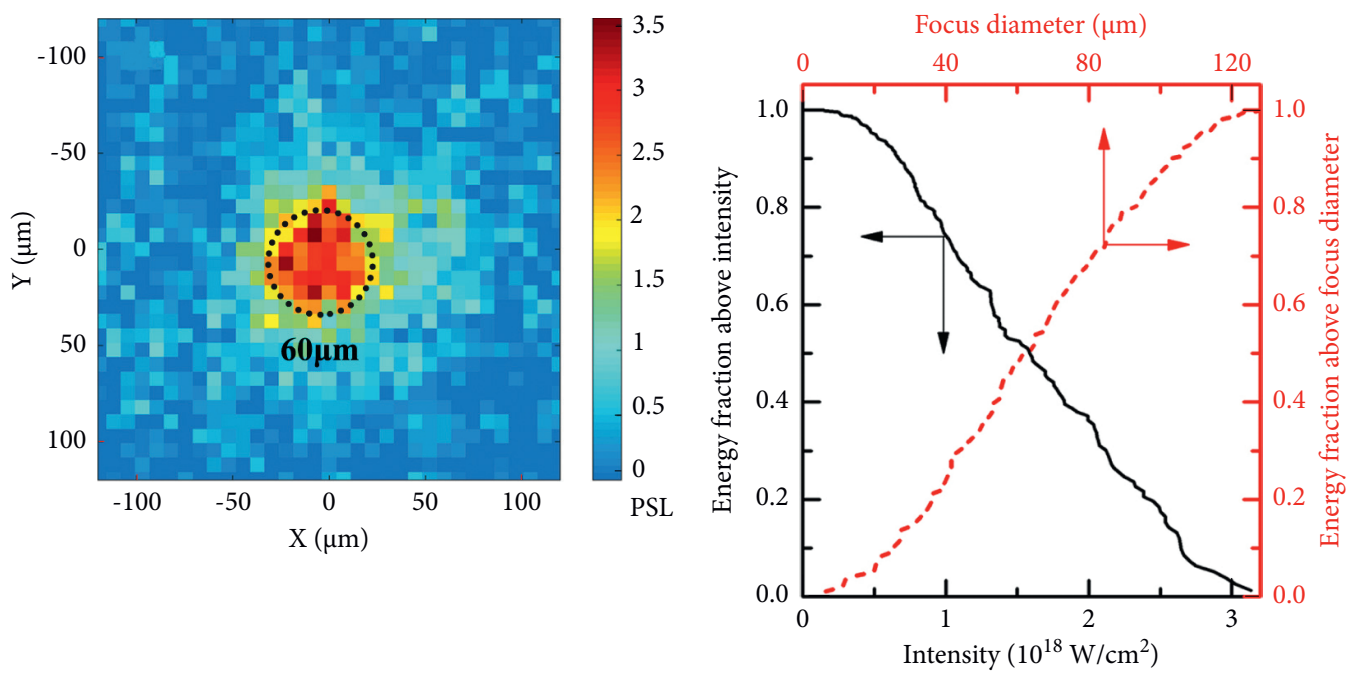

(a)

(b)

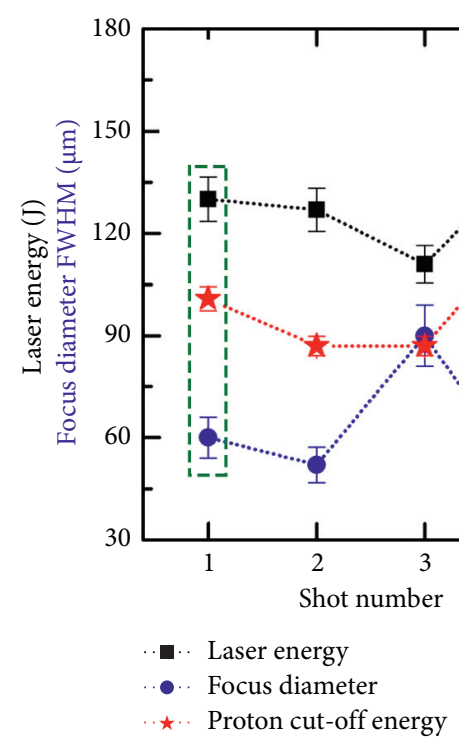

(c)

Figure 2: (a) A typical laser focus image recorded by an X-ray pinhole camera. PSL is the unit of the signal intensity on the imaging plate. (b) Curves of the cumulative laser energy fraction as functions of laser intensity and focus diameter obtained from (a). (c) Experimental results measured in each shot. The shot number 1 is chosen as the typical shot (data in the green box).

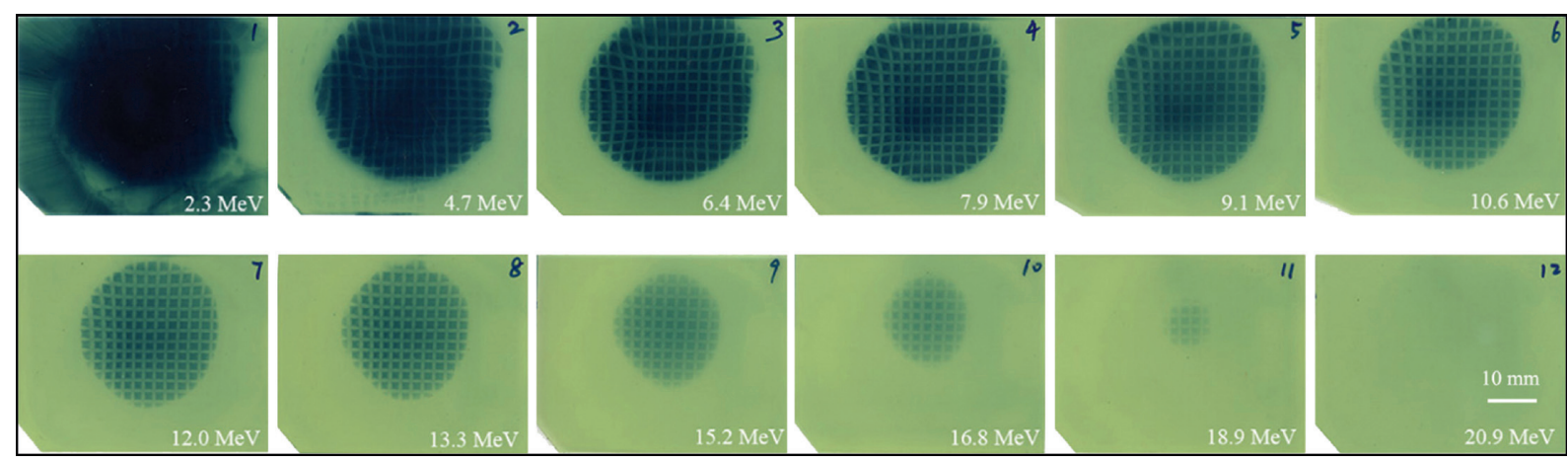

(a)

Figure 3: Continued. 


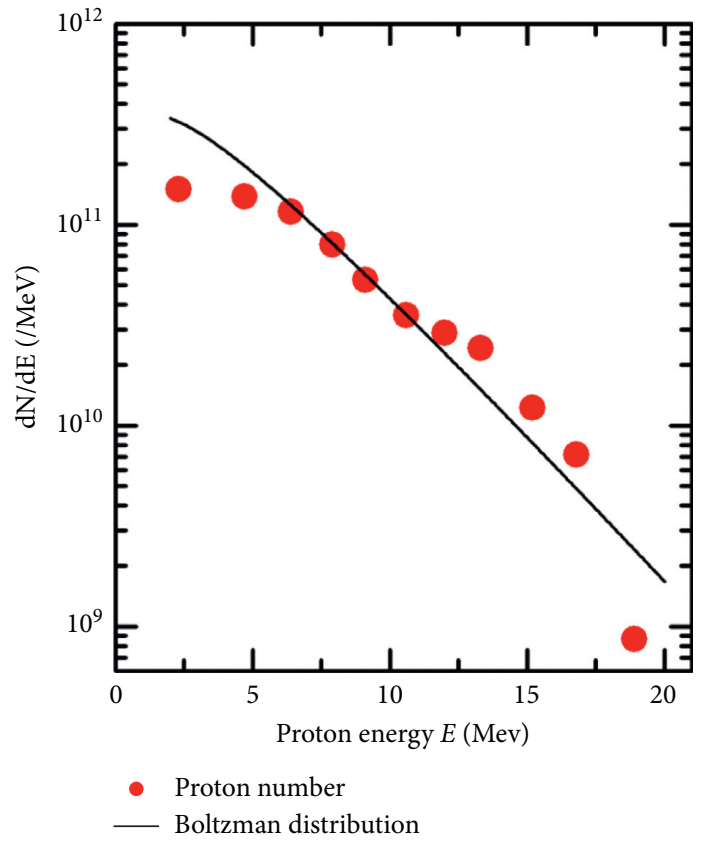

(b)

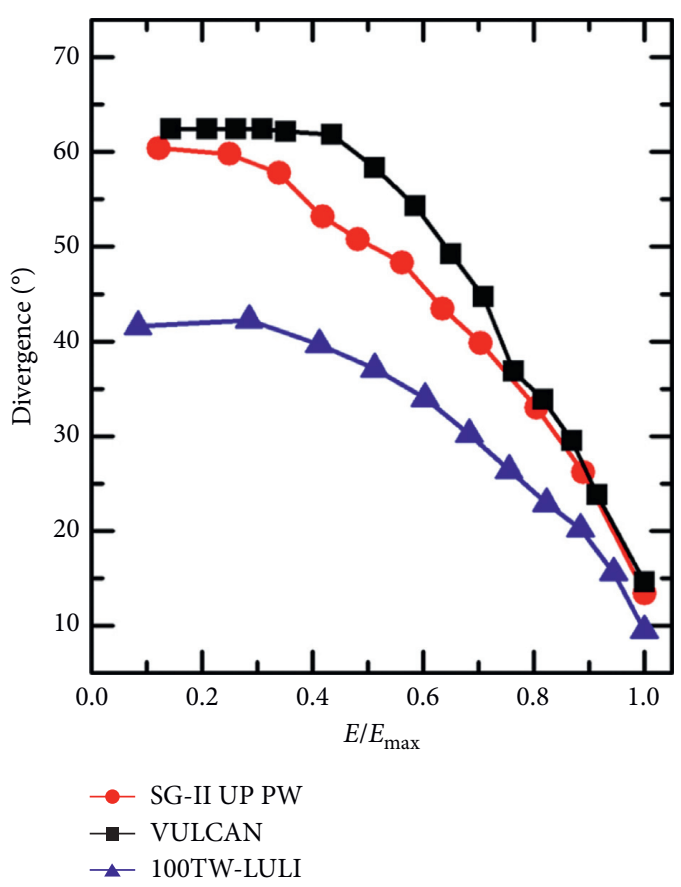

(c)

Figure 3: (a) Proton images on RCF layers for the same shot as in Figure 2(a). (b) Proton energy spectrum derived from (a), which was fitted with Boltzmann distribution. (c) Divergence angle of the protons. The results from VULCAN and 100TW-LULI lasers by Nürnberg et al. [26] are used for comparison.

energy, with the divergence angle decreasing with the increase in the proton energy. The divergence angles of energyresolved protons generated by the SG-II UP PW laser and VULCAN laser are similar but relatively differ from the 100 TW-LULI laser. Note that the laser energy for the SG-II UP PW and VULCAN shot is about $130 \mathrm{~J}$ and that for the $100 \mathrm{TW}$-LULI shot is about $15 \mathrm{~J}$. That may be the reason why their divergences of protons are different.

3.2. Proton Source Location and Size. It has been shown that the laser-driven protons are emitted from a virtual source in a quasilaminar fashion [20]. The location of the virtual source can be obtained through calculating the magnification rate $M$ of the $\mathrm{Cu}$ mesh in the proton image. The magnification rate $M$ of the point-projection imaging in our experiment is the ratio of the virtual source-to-RCF layer distance to the virtual source-to-mesh distance.

$$
M=\frac{(\nu+d+L+l)}{(\nu+d)},
$$

where $v$ is the distance from the virtual source to the foil target and $l$ is the distance from the front surface of the RCF stack to a RCF layer with specific proton energy. In the experiment, the magnification rate $M$ can be measured as $M=d_{\mathrm{RCF}} / d_{\text {mesh }}$, where $d_{\mathrm{RCF}}$ is the period of the mesh in the proton image and $d_{\text {mesh }}$ is the real period of the mesh. For the image of protons with energy of $16.8 \mathrm{MeV}$ shown in Figure $3(\mathrm{a}), d_{\mathrm{RCF}}=2.131$ $\mathrm{mm}, d_{\text {mesh }}=0.126 \mathrm{~mm}, l=2.2 \mathrm{~mm}, d=2 \mathrm{~mm}$, and $L=32$ $\mathrm{mm}$, we can then obtain $v=0.151 \mathrm{~mm}$. Using the same method, the positions of the virtual sources for protons with different energies in front of the target are obtained with the uncertainty of about $7 \%-15 \%$, as listed in Table 1 .

To estimate the size of the virtual source, a series of Monte Carlo simulations based on Geant 4 were carried out for the protons with various source sizes [11, 30]. In the simulations, spatial Gaussian density distribution in the proton source was used.

$$
n(x, y)=n_{0} \exp \left(-\frac{x^{2}}{r^{2}}-\frac{y^{2}}{r^{2}}\right)
$$

where $n_{0}$ and $r$ are the total proton number and the 1/e is the radius of the proton source, respectively. The spatially resolved optical density distribution in the RCF layer is calculated, as shown in Figure 4. By comparing the experimental optical density profile with the simulated ones, we find that the protons with a virtual source size of about $12 \mu \mathrm{m}$ in $1 / e$ diameter can well match the experimental optical density profile.

3.3. Spatial Resolution of the Proton Imaging. The spatial resolution of the proton imaging reflects the size of the proton virtual source. We, therefore, analysed the mesh images on RCFs to obtain the point spread function (PSF) so as to quantify the proton imaging spatial resolution. We first fitted the edge of the experimental optical density profile in Figure 4(b) for $X$ from $11 \mathrm{~mm}$ to $14 \mathrm{~mm}$ with an edge spread function (ESF) [31], which is 
TABLE 1: Energy-resolved position of the virtual source in front of the target $\nu$ and virtual source size $S_{\text {virtual }}$ (for VULCAN and 100TW-LULI lasers) or $2 \sigma$ of ESF value (for SG-II UP PW) for three different laser systems. The results from VULCAN and 100TW-LULI lasers by Nürnberg et al. [26] are used for comparison.

\begin{tabular}{lccc}
\hline Laser system & $E(\mathrm{MeV})$ & $\nu(\mu \mathrm{m})$ & $S_{\text {virtual }}$ or $2 \sigma$ of ESF $(\mu \mathrm{m})$ \\
\hline & 6.4 & 418 & 19.2 \\
& 7.9 & 392 & 22.9 \\
& 9.1 & 381 & 13.7 \\
SG-II UP PW & 10.6 & 306 & 12.4 \\
& 12.0 & 268 & 10.2 \\
& 13.3 & 231 & 10.7 \\
& 15.2 & 179 & 11.0 \\
\hline \multirow{2}{*}{ VULCAN } & 16.8 & 89 & 13.8 \\
\hline \multirow{2}{*}{ 100TW-LULI } & 18.9 & 372 & 17.9 \\
& 6.2 & 173 & 12.1 \\
& 17.4 & 75 & 11.0 \\
\hline
\end{tabular}

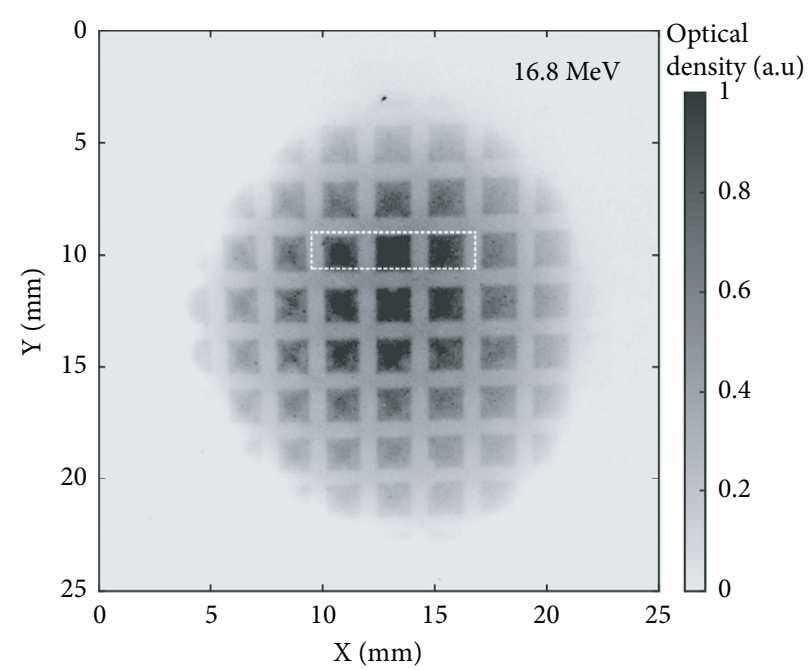

(a)

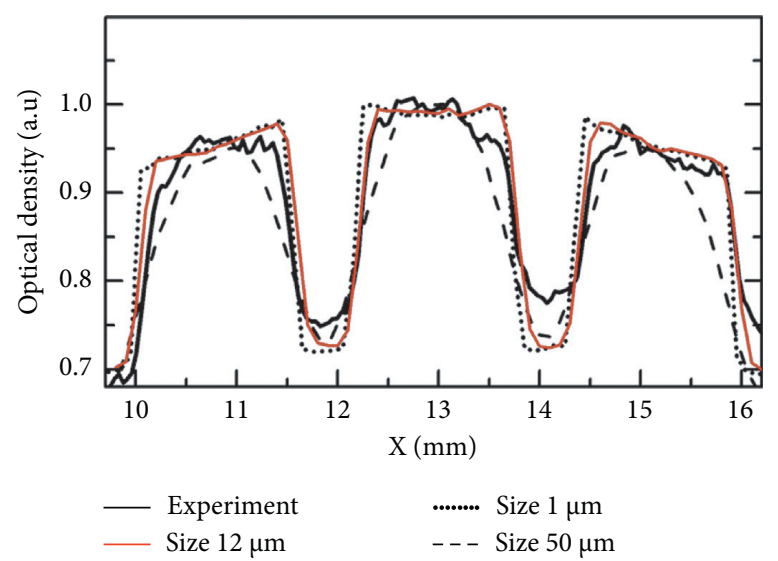

(b)

Figure 4: (a) The optical density distribution of $16.8 \mathrm{MeV}$ protons in Figure 3(a). (b) The experimental optical density profile of $16.8 \mathrm{MeV}$ protons in the white box region in (a), and the simulated optical density profile of $16.8 \mathrm{MeV}$ protons with source sizes of $1 \mu \mathrm{m}, 12 \mu \mathrm{m}$, and $50 \mu \mathrm{m}$ in $1 / e$ diameter, respectively.

$$
I_{\mathrm{ESF}}(x)=I_{0} \cdot \operatorname{erf}\left(\frac{x}{\sigma}\right)+a_{0}+a_{1} x
$$

where

$$
\operatorname{erf}\left(\frac{x}{\sigma}\right)=\frac{2}{\pi} \int_{0}^{x / \sigma} e^{-t^{2}} \mathrm{~d} t
$$

and $I_{0}, \sigma, a_{0}$, and $a_{1}$ are fitting coefficients. The fitted ESF is shown in Figure 5(a), which gives $\sigma=6.9 \mu \mathrm{m}$. Considering that the ESF is the integral response function of a Gaussiantype PSF, the PSF can be obtained as

$$
I_{\mathrm{PSF}}(x)=I_{0} \frac{2}{\pi \sigma} \exp \left(\frac{-x^{2}}{\sigma^{2}}\right)+a_{1} .
$$

So the full width at 1/e maximum of spatial resolution of proton imaging is $2 \sigma=13.8 \mu \mathrm{m}$. This is consistent with the size of the proton virtual source of about $12 \mu \mathrm{m}$ obtained through Monte Carlo simulations. The energy-resolved $2 \sigma$ of ESF values are listed in Table 1 and are compared with the virtual source sizes from VULCAN and 100TW-LULI lasers by Nürnberg et al. [26].

In addition, the normalized Fourier transform of the PSF represents the modulation transfer function (MTF), which is

$$
I_{\mathrm{MTF}}(k)=\exp \left(-\frac{k^{2} \sigma^{2}}{4}\right),
$$

where $k=2 \pi / \lambda$ and $\lambda$ is the period of the modulation. Figure 5(b) shows the MTF for the protons with different energies. One sees that the spatial resolution of the $16.8 \mathrm{MeV}$ proton imaging at $20 \% \mathrm{MTF}$ is about $15 \mu \mathrm{m}$ and that for other protons ranges from 12 to $25 \mu \mathrm{m}$. 


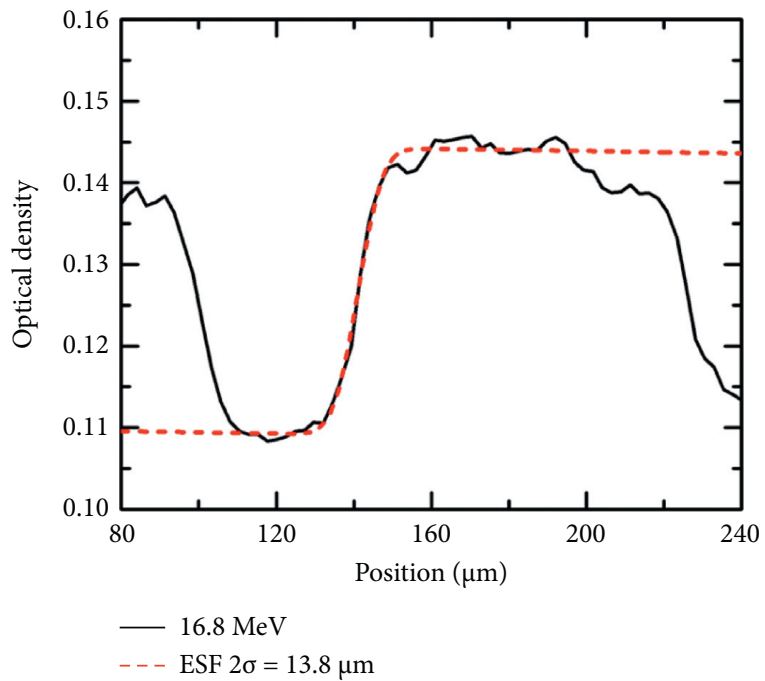

(a)

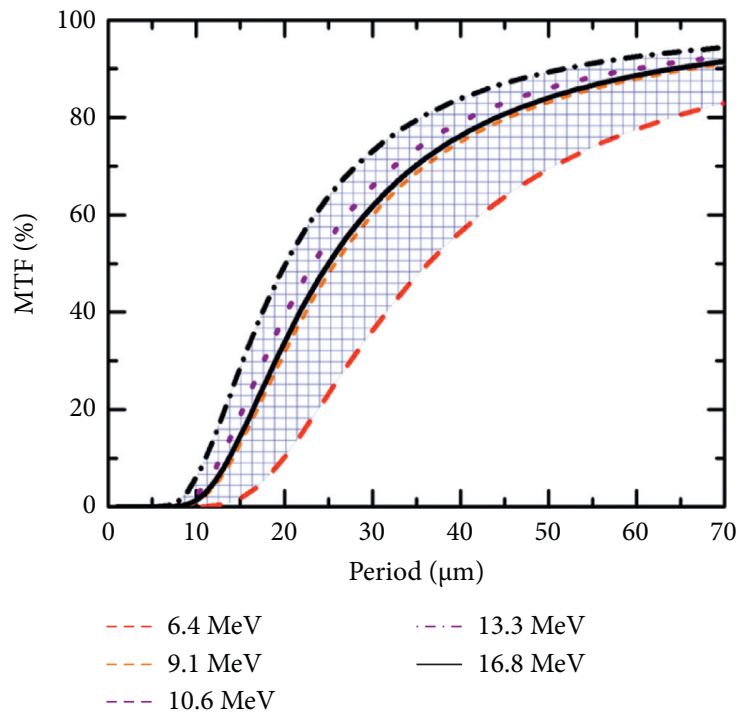

(b)

Figure 5: (a) The experimental optical density profile in Figure 4(b) for $X$ from $11 \mathrm{~mm}$ to $14 \mathrm{~mm}$ was fitted by an edge spread function (ESF) with the coefficient $2 \sigma=13.8 . M=d_{\mathrm{RCF}} / d_{\text {mesh }}=16.9 \mathrm{M}=\left(d_{\mathrm{RCF}}\right) /\left(d_{\text {mesh }}\right)=16.9$ is the magnification rate of the imaging for the protons with energy of $16.8 \mathrm{MeV}$. (b) The MTF for the protons with different energies; the shaded area represents the range of the possible MTF.

\section{Conclusions}

In summary, the characteristics of the energetic protons accelerated from the SG-II UP PW laser interactions with foil targets were investigated. The maximum proton energy obtained in the experiment was $18.9 \mathrm{MeV}$ with a laser pulse of $130 \mathrm{~J}$ and $1 \mathrm{ps}$. The energy spectrum of protons measured by the RCF stack is fitted with the Boltzmann distribution with the temperature of about $2.8 \mathrm{MeV}$. The emission of the protons showed a decreasing divergence angle from $60^{\circ}$ to $10^{\circ}$ with the increase in the proton energy. Based on the proton point-projection mesh imaging, the proton virtual sources for protons with different energies were found to locate tens to hundreds of microns in front of the target, and the size was estimated with a Monte Carlo simulation, to be about $12 \mu \mathrm{m}$ in 1/e diameter for the protons with energy of $16.8 \mathrm{MeV}$. This size is consistent with the proton imaging spatial resolution of about $15 \mu \mathrm{m}$ obtained from the point spread function. These results are of importance for experiments using the protons as a backlighting source on the SG-II UP PW laser.

\section{Data Availability}

Data can be made available upon request.

\section{Conflicts of Interest}

The authors declare no conflicts of interest.

\section{Acknowledgments}

The authors gratefully acknowledge the support from the technical team of the ShenGuang-II laser facility. This work was supported by the National Science and Technology
Innovation Foundation of the Chinese Academy of Sciences (Grant No. CXJJ-20S015) and the Strategic Priority Research Program of the Chinese Academy of Sciences (Grant No. XDA 25020204).

\section{References}

[1] E. L. Clark, K. Krushelnick, J. R. Davies et al., "Measurements of energetic proton transport through magnetized plasma from intense laser interactions with solids," Physical Review Letters, vol. 84, no. 4, pp. 670-673, 2000.

[2] R. A. Snavely, M. H. Key, S. P. Hatchett et al., "Intense highenergy proton beams from Petawatt-laser irradiation of solids," Physical Review Letters, vol. 85, no. 14, pp. 2945-2948, 2000.

[3] S. P. Hatchett, C. G. Brown, T. E. Cowan et al., "Electron, photon, and ion beams from the relativistic interaction of Petawatt laser pulses with solid targets," Physics of Plasmas, vol. 7, no. 5, pp. 2076-2082, 2000.

[4] A. Pukhov, "Three-dimensional simulations of ion acceleration from a foil irradiated by a short-pulse laser," Physical Review Letters, vol. 86, no. 16, pp. 3562-3565, 2001.

[5] H. Daido, M. Nishiuchi, and A. S. Pirozhkov, "Review of laserdriven ion sources and their applications," Reports on Progress in Physics, vol. 7, Article ID 056401, 2012.

[6] S. C. Wilks, A. B. Langdon, T. E. Cowan et al., "Energetic proton generation in ultra-intense laser-solid interactions," Physics of Plasmas, vol. 8, no. 2, pp. 542-549, 2001.

[7] C. A. Cecchetti, M. Borghesi, J. Fuchs et al., "Magnetic field measurements in laser-produced plasmas via proton deflectometry," Physics of Plasmas, vol. 16, Article ID 043102, 2009.

[8] A. Chien, L. Gao, H. Ji et al., "Study of a magnetically driven reconnection platform using ultrafast proton radiography," Physics of Plasmas, vol. 26, Article ID 062113, 2019.

[9] H. Ahmed, S. Kar, G. Cantono et al., "Investigations of ultrafast charge dynamics in laser-irradiated targets by a self probing technique employing laser driven protons," Nuclear 
Instruments and Methods in Physics Research Section A: Accelerators, Spectrometers, Detectors and Associated Equipment, vol. 829, pp. 172-175, 2016.

[10] E. Aktan, H. Ahmed, B. Aurand et al., "Parametric study of a high amplitude electromagnetic pulse driven by an intense laser," Physics of Plasmas, vol. 26, Article ID 070701, 2019.

[11] M. Roth, T. E. Cowan, M. H. Key et al., "Fast ignition by intense laser-accelerated proton beams," Physical Review Letters, vol. 86, no. 3, pp. 436-439, 2001.

[12] V. Malka, S. Fritzler, E. Lefebvre et al., "Practicability of protontherapy using compact laser systems," Medical Physics, vol. 31, no. 6, pp. 1587-1592, 2004.

[13] U. Masood, M. Bussmann, T. E. Cowan et al., "A compact solution for ion beam therapy with laser accelerated protons," Applied Physics B, vol. 117, no. 1, pp. 41-52, 2014.

[14] P. Chaudhary, G. Milluzzo, H. Ahmed et al., "Radiobiology experiments with ultra-high dose rate laser-driven protons: methodology and state-of-the-art," Frontiers in Physics, vol. 9, Article ID 624963, 2021.

[15] T. F. Rösch, Z. Szabó, D. Haffa et al., "A feasibility study of zebrafish embryo irradiation with laser-accelerated protons," The Review of Scientific INSTRUMENTS, vol. 91, Article ID 063303, 2020.

[16] F. Hanton, P. Chaudhary, D. Doria et al., "DNA DSB repair dynamics following irradiation with laser-driven protons at ultra-high dose rates," Scientific Reports, vol. 9, no. 1, p. 4471, 2019.

[17] M. Barberio, S. Giusepponi, S. Vallières, M. Scisció, M. Celino, and P. Antici, "Ultra-fast high-precision metallic nanoparticle synthesis using laser-accelerated protons," Scientific Reports, vol. 10 , no. 1, p. 9570, 2020.

[18] A. Kleinschmidt, V. Bagnoud, O. Deppert et al., "Intense, directed neutron beams from a laser-driven neutron source at PHELIX," Physics of Plasmas, vol. 25, Article ID 053101, 2018.

[19] M. Borghesi, D. H. Campbell, A. Schiavi et al., "Electric field detection in laser-plasma interaction experiments via the proton imaging technique," Physics of Plasmas, vol. 9, no. 5, pp. 2214-2220, 2002.

[20] M. Borghesi, A. J. Mackinnon, D. H. Campbell et al., "Multi$\mathrm{MeV}$ proton source investigations in ultraintense laser-foil interactions," Physical Review Letters, vol. 92, Article ID 055003, 2004.

[21] T. E. Cowan, J. Fuchs, H. Ruhl et al., "Ultralow emittance, multi-MeV proton beams from a laser virtual-cathode plasma accelerator," Physical Review Letters, vol. 92, no. 20, Article ID 204801, 2004.

[22] J. Zhu, J. Zhu, X. Li et al., "Status and development of highpower laser facilities at the NLHPLP," High Power Laser Science and Engineering, vol. 6, p. e55, 2018.

[23] S. N. Chen, M. Gauthier, M. Bazalova-Carter et al., "Absolute dosimetric characterization of Gafchromic EBT3 and HDv2 films using commercial flat-bed scanners and evaluation of the scanner response function variability," Review of Scientific Instruments, vol. 87, Article ID 073301, 2016.

[24] C. Wang, H. H. An, J. Xiong et al., "A pinhole camera for ultrahigh-intensity laser plasma experiments," Review of Scientific Instruments, vol. 88, no. 11, Article ID 113501, 2017.

[25] W. Theobald, C. Sorce, W. R. Donaldson et al., "Inferred UV fluence focal-spot profiles from soft $\mathrm{x}$-ray pinhole-camera measurements on OMEGA," The Review of Scientific Instruments, vol. 91, Article ID 023505, 2020.

[26] F. Nürnberg, M. Schollmeier, E. Brambrink et al., "Radiochromic film imaging spectroscopy of laser-accelerated proton beams," The Review of Scientific Instruments, vol. 80, Article ID 033301, 2009.

[27] D. S. Hey, M. H. Key, A. J. Mackinnon et al., "Use of GafChromic film to diagnose laser generated proton beams," Review of Scientific Instruments, vol. 7, Article ID 053501, 2008.

[28] J. F. Ziegler, M. D. Ziegler, and J. P. Biersack, "SRIM-the stopping and range of ions in matter (2010)," Nuclear Instruments and Methods in Physics Research Section B: Beam Interactions with Materials and Atoms, vol. 268, no. 11-12, pp. 1818-1823, 2010.

[29] M. Schollmeier, M. Geissel, A. B. Sefkow, and K. A. Flippo, "Improved spectral data unfolding for radiochromic film imaging spectroscopy of laser-accelerated proton beams," The Review of Scientific Instruments, vol. 85, Article ID 043305, 2014.

[30] S. Incerti, G. Baldacchino, M. Bernal et al., "The Geant4-DNA project," International Journal of Modeling, Simulation, and Scientific Computing, vol. 1, no. 2, pp. 157-178, 2010.

[31] H.-S. Park, B. R. Maddox, E. Giraldez et al., "High-resolution 17-75 keV backlighters for high energy density experiments," Physics of Plasmas, vol. 15, Article ID 072705, 2008. 